\title{
Tumor-suppressive mir-663 gene induces mitotic catastrophe growth arrest in human gastric cancer cells
}

\author{
JIAN PAN, HAI HU, ZHUAN ZHOU, LICHAO SUN, LIANG PENG, LONG YU, \\ LIXIN SUN, JUN LIU, ZHIHUA YANG and YULIANG RAN \\ The State Key Laboratory of Molecular Oncology, Cancer Institute (Hospital), Chinese Academy of \\ Medical Sciences, Peking Union Medical College, Beijing 100021, P.R. China
}

Received December 2, 2009; Accepted March 5, 2010

DOI: 10.3892/or_00000834

\begin{abstract}
Increasing evidence suggests that microRNAs are involved in human carcinogenesis as tumor suppressors or oncogenes. A growing number of reports has shown that an interest has been sparked in aberrant microRNA expression and how they can be used to treat human diseases, including cancer. However, their precise biological role remains largely unknown. In the present study, we aimed to identify microRNA species involved in the regulation of tumor growth. By performing quantitative reverse transcription-polymerase chain reaction (RT-PCR) analysis, we demonstrated that mir-663 was downregulated in human gastric cancer cell lines unlike in normal cells. A transient introduction of mir-663 into the human gastric cancer cell lines BGC823 and SNU5 induced morphology changes and suppression of proliferation of these cells. In addition, mir-663 alters the DNA content and induces phenotypes of mitotic catastrophe in tumor cells. Moreover, the liposome-mediated delivery of mir-663 suppressed the in vivo growth of the BGC823 and SNU5 cells. Western blot analyses performed after the introduction of mir-663 revealed upregulation of cyclin B following transfection with mir-663. Our results provide evidence that downregulation of mir-663 in tumor cells may contribute to aberrant cell hyperplasia, leading to the development of gastric cancer. Therefore, mir-663 might function as a potent suppressor of tumor growth.
\end{abstract}

\section{Introduction}

The known classes of genes that function as tumor suppressors and oncogenes have recently been expanded to include the microRNA (miRNA) family of regulatory molecules. miRNAs are a class of conserved non-coding 20-22-nt small RNAs that regulate gene expression by binding to mRNA; this binding leads to mRNA degradation or inhi-

Correspondence to: Dr Yuliang Ran, The State Key Laboratory of Molecular Oncology, Cancer Institute (Hospital), Chinese Academy of Medical Sciences, Peking Union Medical College, Beijing 100021, P.R. China

E-mail: ran_yuliang@yahoo.com.cn

Key words: mir-663, gastric cancer, mitotic catastrophe bition (1). miRNAs have been implicated in diverse processes such as cellular differentiation, cell cycle control, and apoptosis (2-6). The analysis of miRNA expression profiles in cancer cells has revealed essentially ubiquitous dysregulation of these molecules in a wide variety of tumors $(1,7-10)$. It is clear that many of these changes in expression are not simply a secondary consequence of the transformation process. Loss of function of specific miRNAs appears to be a key event in the genesis of diverse cancers. The loss of function of miR-15a and miR-16-1 due to genomic deletion is very common in chronic lymphocytic leukemia (CLL), mantle cell lymphoma (MCL), multiple myeloma, and prostate cancer (11). The downregulation of the let-7 family, the gene clusters of at least 4 of which are found in regions frequently deleted in diverse human malignancies, is associated with poor prognosis in lung cancer $(2,12)$. Since many classical tumor suppressor genes are inactivated not only by deletion or mutation but also by aberrant hypermethylation, we hypothesized that miRNA genes might be silenced in human tumors by the hypermethylation of $\mathrm{CpG}$ islands surrounding them $(13,14)$. Mir-663 is located in a hypothetic gene and has a $\mathrm{CpG}$ island near the promoter. In a study, aberrant hypermethylation was found in $62 \%$ of 71 primary human breast cancer specimens (15). However, the role of mir-663 in cancer development remains largely unknown.

In this study, we found that mir-663 was downregulated in human gastric cancer cell lines. Transient introduction of mir-663 into the human gastric cancer cell lines BGC823 and SNU5 induces phenotypes of mitotic catastrophe in tumor cells, but not in normal cells. miRNA-based treatment for human tumors has been documented extensively. The tumor suppressor role of mir-34 has been confirmed in vivo with these tumor xenografts via activation of the p53 pathway (16). The use of locked nucleic acid anti-mir-21 oligonucleotides has been reported to reduce the growth of U87 glioblastoma xenograft by $>70 \%$ (17). In the present study, mir-663 significantly suppressed the in vivo growth of BGC823 and SNU5 cells when introduced into the cells by lipid-mediated gene delivery. There is currently an increased interest in using miRNAs for therapeutic benefit.

\section{Materials and methods}

Cell culture conditions and transfection. The gastric epithelial cell line GES1 (a gift from Dr Yang Ke, Peking 
University Medical School, Beijing 100021, China) was cultured in RPMI-1640 supplemented with $10 \%$ fetal bovine serum (FBS). Other cell lines (HEK293, HUVEC, BGC823, SNU5, SNU16, MKN45, and N87) were purchased from the American Type Culture Collection (ATCC). The cells were transfected $24 \mathrm{~h}$ after being seeded in 6-well plates. The miRNA mir-663 mimic RNA 5'-AGGCGGGGCGCCGCG GGACCGC-3' and a random mimic RNA 5'-GGCGGGA GGCGCGCCGCAGGCC-3' (100 p mol; synthesized by Ribobio Inc., Guangzhou, China) was added to $200 \mu \mathrm{l}$ of serum-free, antibiotic-free medium and mixed with $5 \mu 1$ of lipofectamine 2000 transfection reagent (Invitrogen, Carlsbad, CA, USA) that was dissolved in $200 \mu 1$ of the same medium and allowed to stand at room temperature for $20 \mathrm{~min}$. The resulting $400 \mu \mathrm{l}$ of the transfection solution was then added to each well containing $1.6 \mathrm{ml}$ of the medium. Six hours later, the cultures were replenished with $2 \mathrm{ml}$ fresh medium supplemented with $10 \%$ FBS and antibiotics. For Western blot analysis, assays of cell proliferation and cell cycle, cells were collected after an additional $72 \mathrm{~h}$.

Quantitative reverse-transcription PCR for mir-663. Quantitative real-time PCR was performed to determine the expression levels of mir-663 genes. Total RNA was reverse transcribed using the Reverse Transcription Kit, according to the manufacturer's protocol (Applied Biosystems Inc., Foster City, CA). The RT primer of U6 was 5'-CGCT TCACGAATTTGCGTGTCAT-3', and the RT primer of mir-663 was 5'-GTCGTATCCAGTGCGTGTCGTGG AGTCGGCAATTGCACTGGATACGACGCGGTCC-3'. The expression was quantified using the PCR primer of U6 (F: 5'-GCTTCGGCAGCACATATACTAAAAT-3', R: 5'-CGCTTCACGAATTTGCGTGTCAT-3') and the PCR primer of mir-663 (F: 5'-GTGCGTGTCGTGGAG TCG-3', R: 5'-TTTAGGCGGGGCG-3'). The expression was normalized using endogenous U6 as the control. The data were analyzed using the SDS relative quantification software (Applied Biosystems Inc.).

Cell proliferation assay. The cells were trypsinized and then resuspended in complete medium and seeded onto 96well plates at $2 \times 10^{3}$ cells per well. The cells were counted by using the cholecystokinin octapeptide (CCK-8) on the indicated days according to the manufacturer's instructions; the optical density (OD) was $450 \mathrm{~nm}$. All experiments were performed in triplicate and repeated twice.

Cell cycle analysis. For cell cycle analysis, cell suspensions were washed twice in phosphate-buffered saline (PBS) and fixed in cold $70 \%$ ethanol for $4 \mathrm{~h}$. The fixed cells were then washed with PBS, incubated with $100 \mathrm{mg} / \mathrm{ml}$ Rnase A for $30 \mathrm{~min}$ at room temperature, stained with propidium iodide (PI) $(25 \mathrm{mg} / \mathrm{ml})$, and analyzed by flow cytometry.

Determination of mitotic catastrophe index. The cells were harvested for the determination of the mitotic index as described previously (18). The slides were examined under a microscope; mir-663-treated cells stained with Giemsa were subdivided into 2 types of cells according to their nuclear morphology. Only cells with distinct interphase nuclei, meta- phase spreads, or the appearance of mitotic catastrophe were counted. Giant cells containing multiple evenly stained nuclei and/or small nuclear fragments (micronuclei) were considered to have an 'abnormal' nucleus. At least 1000 cells were counted on each slide for the determination of the mitotic catastrophe index. Three independent experiments were analyzed (19).

Chromosome analysis. After 72 h of culture incubation, $0.25 \mathrm{ml}$ colchicine $(10 \mu \mathrm{g} / \mathrm{ml})$ was added to each $15-\mathrm{ml}$ tube. The tubes were inverted several times to ensure proper mixing. Next, the cultures were returned to the incubator at $37^{\circ} \mathrm{C}$ for $20 \mathrm{~min}$. The cells were resuspended by inverting the tubes, and then the tubes were centrifuged for $8 \mathrm{~min}$ at $1200 \mathrm{rpm}$. The supernatant was aspirated with a sterile Pasteur pipette connected to a vacuum apparatus. The cells were resuspended by using a bulbed 9 inch Pasteur pipette. A warm hypotonic $\mathrm{KCl}$ solution (1-3 ml) was slowly added to the tubes until a volume of $10 \mathrm{ml}$ of $0.075 \mathrm{M} \mathrm{KCl}$ was reached. Air was blown into the suspension with a bulbed pipette after each addition of the $\mathrm{KCl}$ solution. Next, the tubes were incubated at $37^{\circ} \mathrm{C}$ for $18 \mathrm{~min}$. Freshly prepared cold fixative $\left[1 \mathrm{ml} ; 1: 3\right.$ methanol: acetic acid $\left.\left(4^{\circ} \mathrm{C}\right)\right]$ was added to each tube very slowly (dropwise). The cells were resuspended in the solution. Next, microslides were prepared by dropping the harvested metaphases onto their surfaces, which were previously rinsed with absolute ethanol. These cells were then stained with Giemsa, and the chromosomes were counted under a microscope.

Immunofluorescence. Antibodies to $\alpha$-tubulin (clone DM1A) and $\mathrm{Cy} 3$-conjugated goat anti-mouse antibodies were purchased from Sigma. Cells were fixed in $2 \%$ formaldehyde for $10 \mathrm{~min}$ and permeabilized with $0.5 \%$ Nonidet P-40 in PBS ( $\alpha$-tubulin). Antibodies to $\alpha$-tubulin were used at a 1:100 dilution. The nuclei were stained with $1 \mathrm{mg} / \mathrm{ml} \mathrm{4',6-diamidino-}$ 2-phenylindole (DAPI), and the cells were analyzed under a Nikon microscope. The nuclear morphology of at least 200 cells in each sample was examined.

Western blotting. For the Western blot analysis, cellular proteins were extracted in $40 \mathrm{mM}$ Tris- $\mathrm{HCl}(\mathrm{pH}$ 7.4) containing $150 \mathrm{mM} \mathrm{NaCl}$ and $1 \%(\mathrm{v} / \mathrm{v})$ Triton $\mathrm{X}-100$, supplemented with a cocktail of protease inhibitors. Equal amounts of protein were resolved on $12 \%$ sodium dodecyl sulfatepolyacrylamide gel electrophoresis (SDS-PAGE) gels and then transferred to a polyvinylidene fluoride (PVDF) membrane. After blocking with 5\% non-fat milk, the membranes were incubated first with the primary antibody ( $4^{\circ} \mathrm{C}$, overnight), cyclin B (PharMingen), surviving cyclindependent kinase 2 (CDK2) (Santa Cruz), cyclin-dependent kinase 4 (CDK4; Santa Cruz), and ß-actin (Sigma). Next, the membranes were incubated with horseradish peroxidase (HRP)-conjugated sheep anti-rabbit or anti-mouse IgG secondary antibodies (Vector, Burlingame, CA). After washing the membranes, the blots were developed using the Super Enhanced chemiluminescence detection kit (Applygen Technologies Inc., Beijing, China). The protein bands were visualized after exposing the membranes to Kodak X-ray film. 

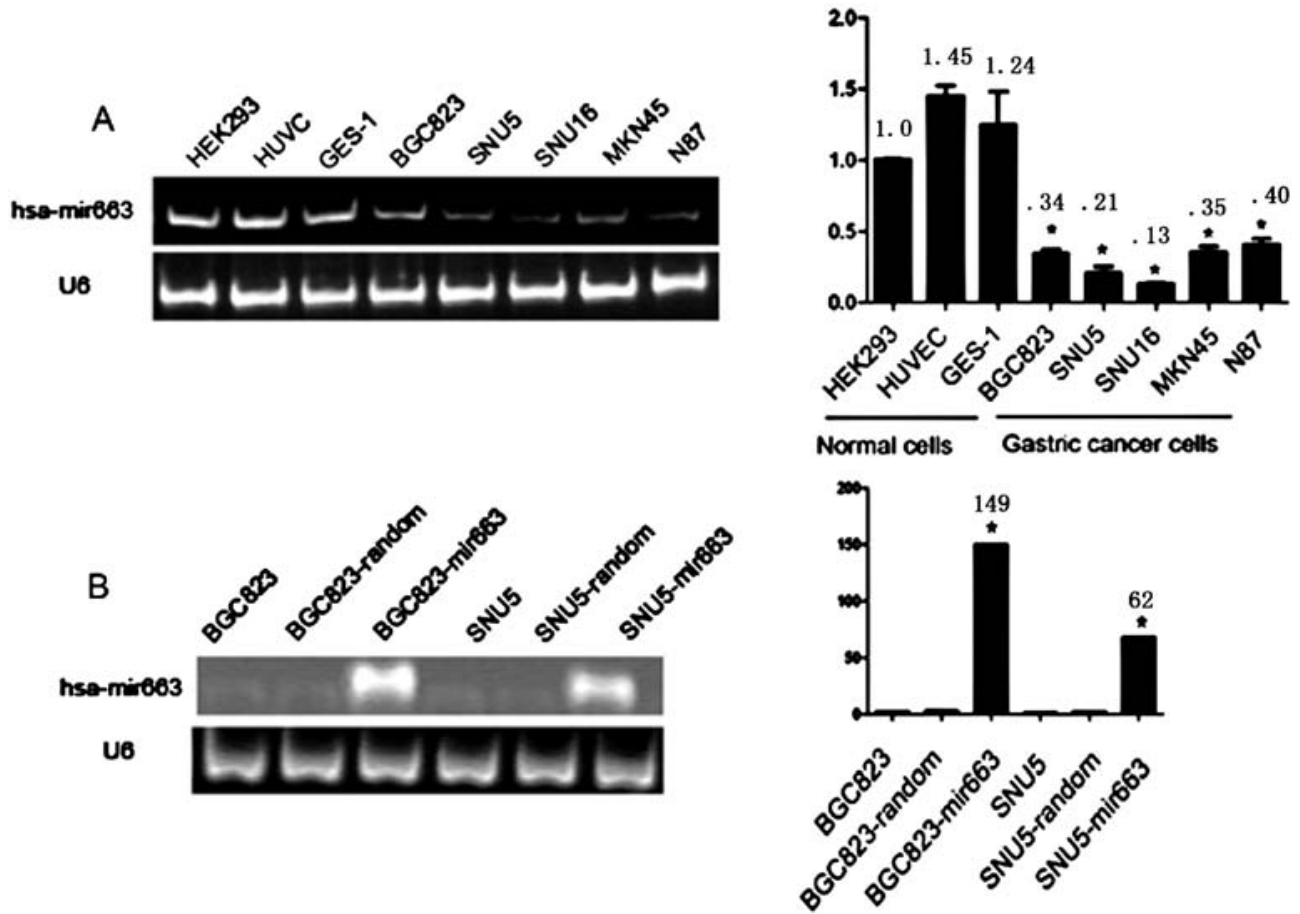

C
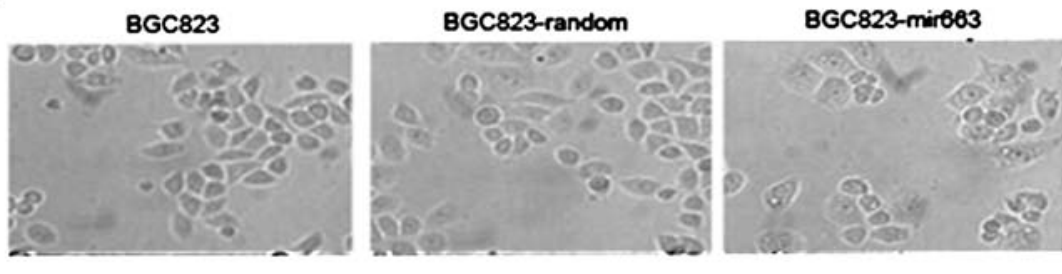

SNU5-paront

SNUS-random

SNU5-mire63
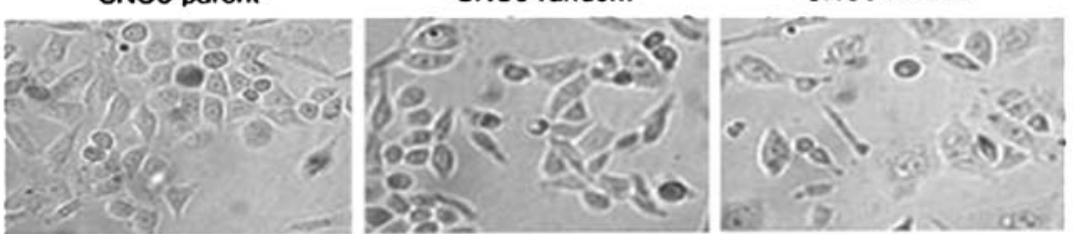

Figure 1. Mir-663 is lowly expressed in gastric carcinoma cell lines and can change the modality of these cells. (a) qRT-PCR analysis of mir-663 expression in 5 GC lines and the normal cell line HEK293, HUVEC and GES1. qRT-PCR analysis of the relative expression levels of mir-663 and target genes U6 in human gastric cancer cell lines. The cells were lyzed to extract total RNA for qRT-PCR. Data were normalized to that of U6 and the relative levels are shown. (b) Quantitative real-time PCR shows that restoration of mir-663 by mir-663 mimic transfection $72 \mathrm{~h}$ after mir-663 mimic transfection of BGC823 and SNU5 cells (100 pmol per well in 6-well plates), mir-663 level was measured by qRTPCR with SYBR-Green PCR system (TaqMan). The comparative threshold cycle CT method was used to calculate relative gene expression levels compared with U6, then normalized with the value from Random mimic as 1. (c) Modality analysis of BGC823 and SNU5 cells transferred with mir-663 mimic RNA.

Suppression of tumor growth by liposome-mediated mir-663 transfection. A volume of $2 \mu 1 \mathrm{mir}-663 \mathrm{RNA}(25 \mu \mathrm{g} / \mu \mathrm{l}$; Ribobio Inc.) was incubated with $100 \mu 1$ of liposome (Engreen Biosystem Co., Ltd., 18668-11) at room temperature for $30 \mathrm{~min}$. After incubation, $102 \mu 1 \mathrm{1X}$ PBS was added until a total volume of $204 \mu \mathrm{l}$ was reached; thus, the final RNA concentration was $250 \mu \mathrm{g} / \mathrm{ml}$. This method of RNA-liposome preparation was based on in vitro optimization of BGC823 and SNU5 cells (20). The tumor-bearing mice were matched by size and divided into groups. The weights of the mice varied by $<5 \%$ in each treatment cohort. Treatment began 9 days after the injection of the tumor cells, when the mice received $50 \mu \mathrm{g}$ mir-663 mimic RNA, $50 \mu \mathrm{g}$ random mimic RNA, or dialysis buffer alone. The therapeutic and control doses were systemically administered into the neoplasm once daily for 10 days; this was followed by a twice-weekly administration until the treatment was completed. The tumor volumes were calculated according to the following formula: volume $=$ length $\mathrm{x}$ width ${ }^{2} / 2$. After the last treatment, the mice were sacrificed and the tumor weight was measured. The animal experiments were conducted according to the ethics regulations of our institute.

Immunohistochemistry. The paraffin-embedded xenograft tumor sections or sections of human tissue samples were assayed. For immunohistochemical staining, the sections were dewaxed, rehydrated, and then incubated with $3 \% \mathrm{H}_{2} \mathrm{O}_{2}$ to quench endogenous peroxidase activity. These sections were then blocked, incubated overnight with primary Ki67 and caspase- 3 antibodies (Maixin Bio, CA; $15 \mu \mathrm{g} / \mathrm{ml}$ in 
A
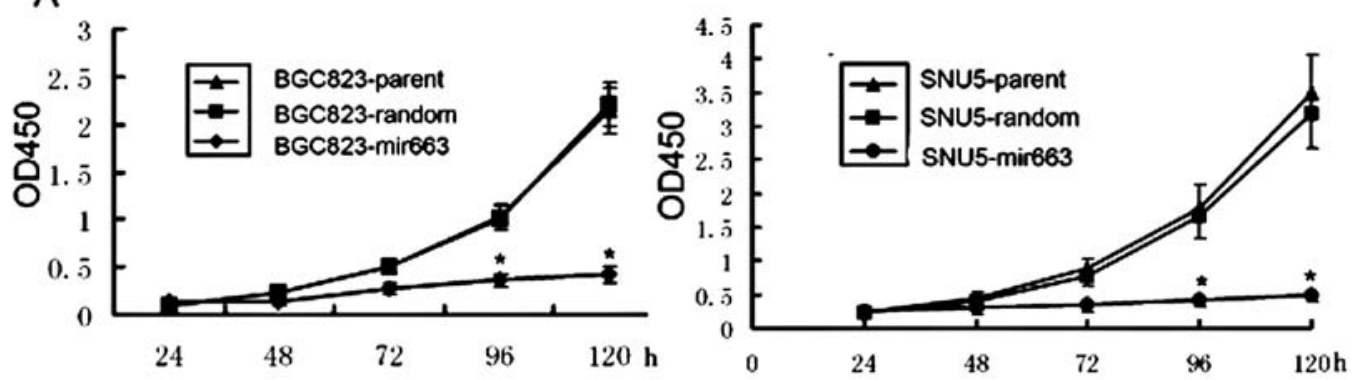

B
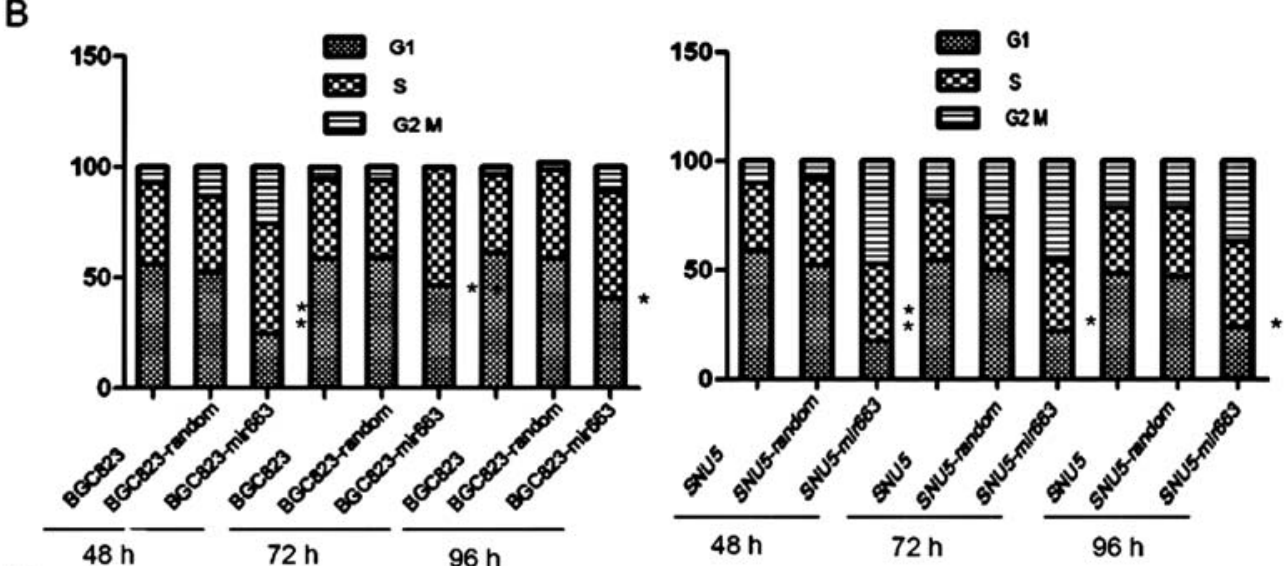

C
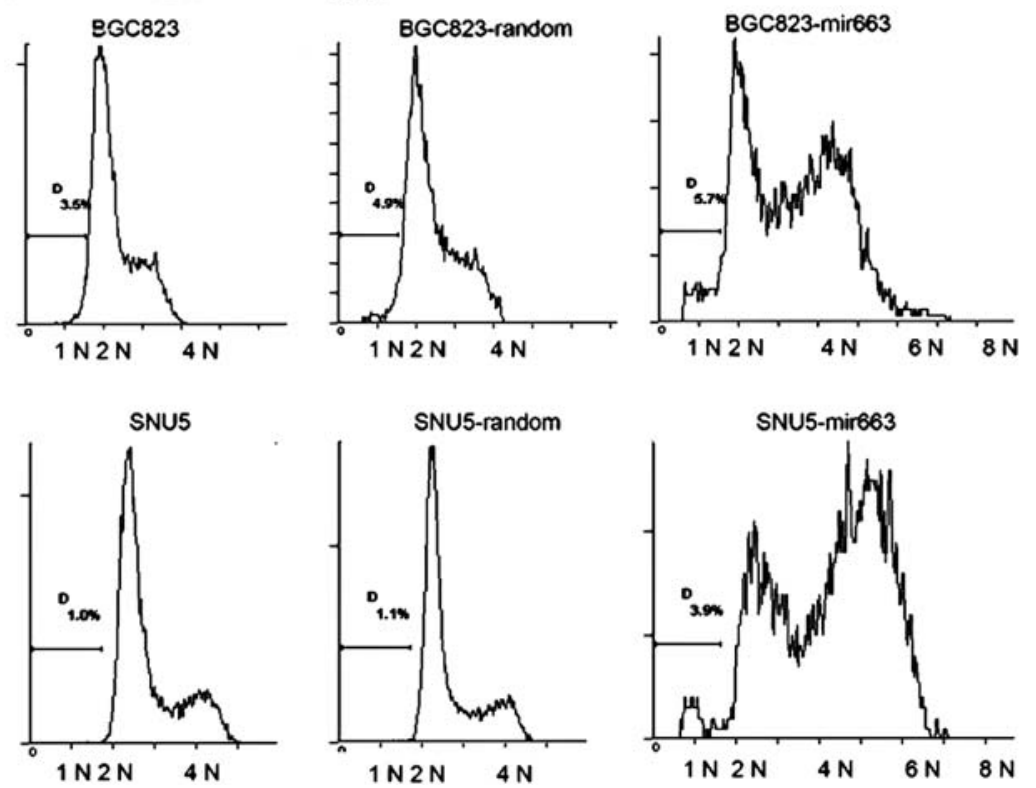

Figure 2. Mir-663 can repress the proliferation of gastric cancer cells and disturb their DNA content. (a) Proliferation analysis of BGC823 and SNU5 cells transferred with mir-663 mimic RNA. (b) Cell cycle analysis of BGC823 and SNU5 cells after mir-663 restoration. Cell cycle analysis was performed $72 \mathrm{~h}$ after transfected with mir-663 mimics or negative control Random mimic. Cells were stained with propidium iodide after ethanol fixation and analyzed by flow cytometry. ${ }^{*} \mathrm{P}<0.05,{ }^{*} \mathrm{P}<0.01$, versus that of Random mimic, one-way ANOVA, $\mathrm{n}=2$. (c) DNA content was disturbed in BGC823 and SNU5 cells after mir-663 restoration. Seventy-two hours after transfection, cells were stained with propidium iodide after ethanol fixation and analyzed by flow cytometry. Relative increase of fluorescence signal was calculated by dividing the normalized signal in each treated sample with that of Random mimic.

PBS) and biotinylated secondary antibody; they were then developed and counterstained with hematoxylin. At least 5 areas of each section were examined at a magnification of $\mathrm{x} 200$.

Statistical analysis. All data are presented as mean \pm SD. Statistical analysis was performed using the Statistical Package for the Social Sciences (SPSS) (SPSS Inc., Chicago, IL).
Student's two-tailed t-test was used to compare the groups. $\mathrm{P} \leq 0.05$ was considered significant.

\section{Results}

The mir-663 gene is expressed in low amounts in gastric cancer cell lines and can change the morphology of these cells. The promoter of mir-663 is reported to be aberrantly hyper- 

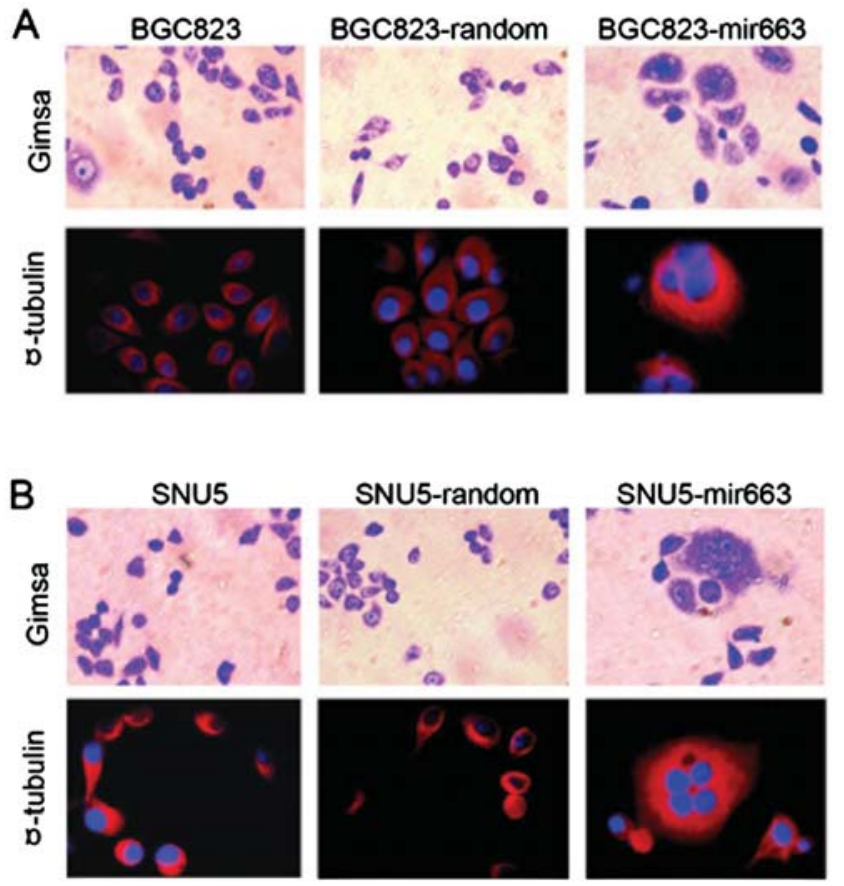

SNU5-mir663

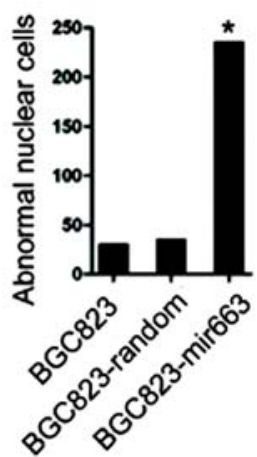

C

D
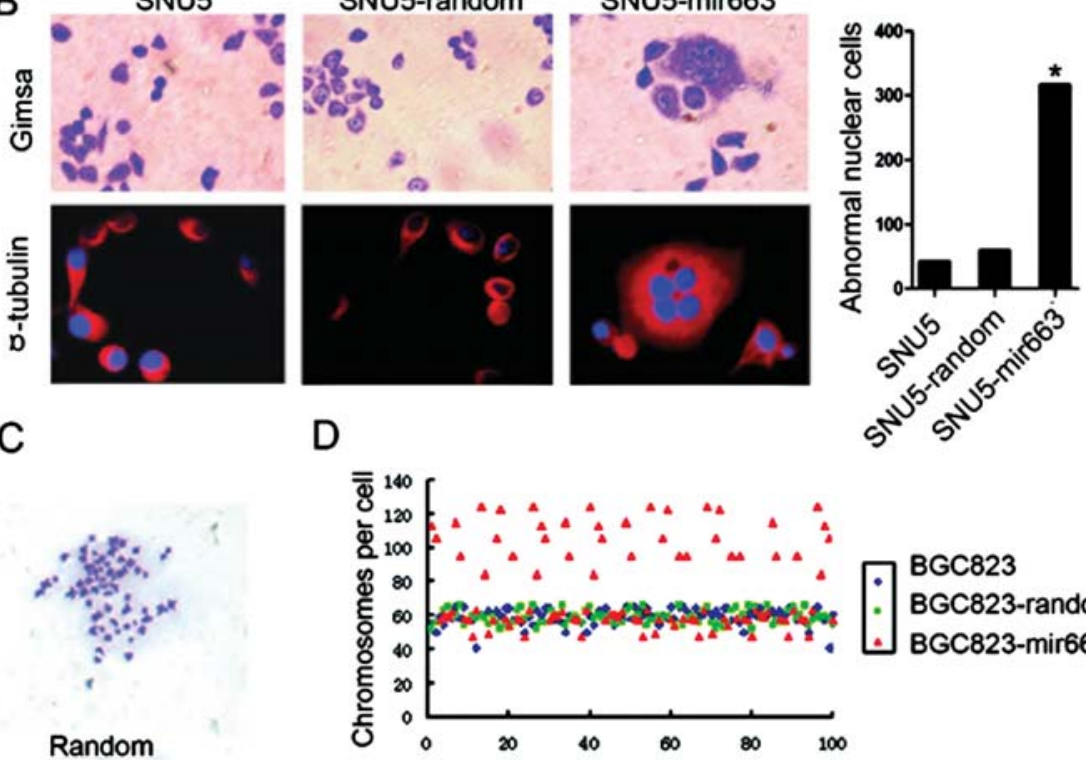

BGC823

BGC823-random

BGC823-mir663

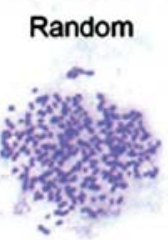

Mir-663

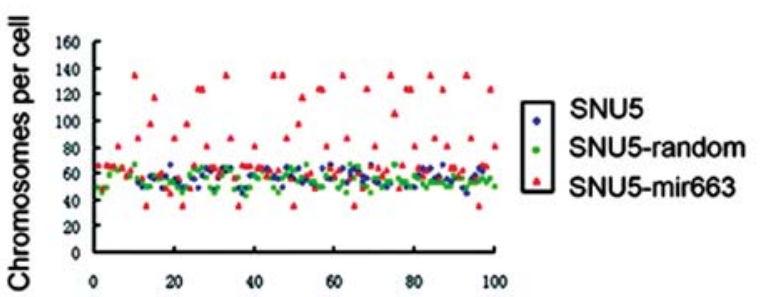

Figure 3. Mir-663 cause mitotic catastrophe of gastric carcinoma cells. (a) Strange structures on the mitotic index slides that stained with Gimsa had more nuclear envelope, it is more legible when cells were stained with DAPI and $\alpha$-tubulin, and these structures were only present in high frequency in mir-663treated cells. (b) One thousand cells per group were examined with microscope. (c) To cells after $72 \mathrm{~h}$ of culture incubation, were added $0.25 \mathrm{ml}$ colchicine $(10 \mu \mathrm{g} / \mathrm{ml})$ with a $1 \mathrm{cc}$ syringe to each $15 \mathrm{ml}$ tube, up to $10 \mathrm{ml}$ of $0.075 \mathrm{M} \mathrm{KCl}$. These cells were stained with Giemsa and the chromosomes were counted with a microscope. (d) Chromosome analysis showed that chromosome number of BGC823 and SNU5 cells transferred with mir-663 was from $<40$ to $>140$, and chromosome number of control group was mostly about 46 .

methylated and might be downregulated in breast cancer cells (15). The RT-PCR data showed that mir-663 was markedly downregulated in gastric cancer cells unlike in human normal gastric epithelial cells (GES1) or HEK293 and HUVEC cells (Fig. 1A). We then performed an in vitro gain-of-function analysis by transfecting the mature miRNAs. At $72 \mathrm{~h}$ post-transfection, we assessed the level of miRNAs by RT-PCR; the mir-663 mimic RNA was successfully transfected into the cancer cells (Fig. 1B). The transfection of the mature mir-663 in BGC823 and SNU5 cells caused a distinct change in these cells. The cells transfected with mir-663 became larger and had multiple modalities (Fig. 1C).

The mir-663 gene suppresses the proliferation of gastric cancer cells and alters their DNA content. The ectopic expression of mir-663 in the BGC823 and SNU5 cell lines resulted in a 5- to 8-fold decrease in cell proliferation (Fig. 2A). The analysis of the cell cycle revealed that mir-663 increased the number of BGC823 and SNU5 cells in the $\mathrm{G} 2 / \mathrm{M}$ phase (Fig. 2B). These results indicated that overexpression of mir-663 is sufficient to decrease the in vitro proliferation of gastric cancer cells. DNA content analysis of these cells revealed that the overexpression of mir-663 altered the DNA content of gastric cancer cells. The BGC823 and SNU5 cells transfected with random mimic RNA had DNA content ranging from 2 to 4 n, and the mir- 663 group had DNA content ranging from 2 to 6 or 8 n (Fig. 2C).

The mir-663 gene causes mitotic catastrophe in gastric carcinoma cells. We observed peculiar structures on the 

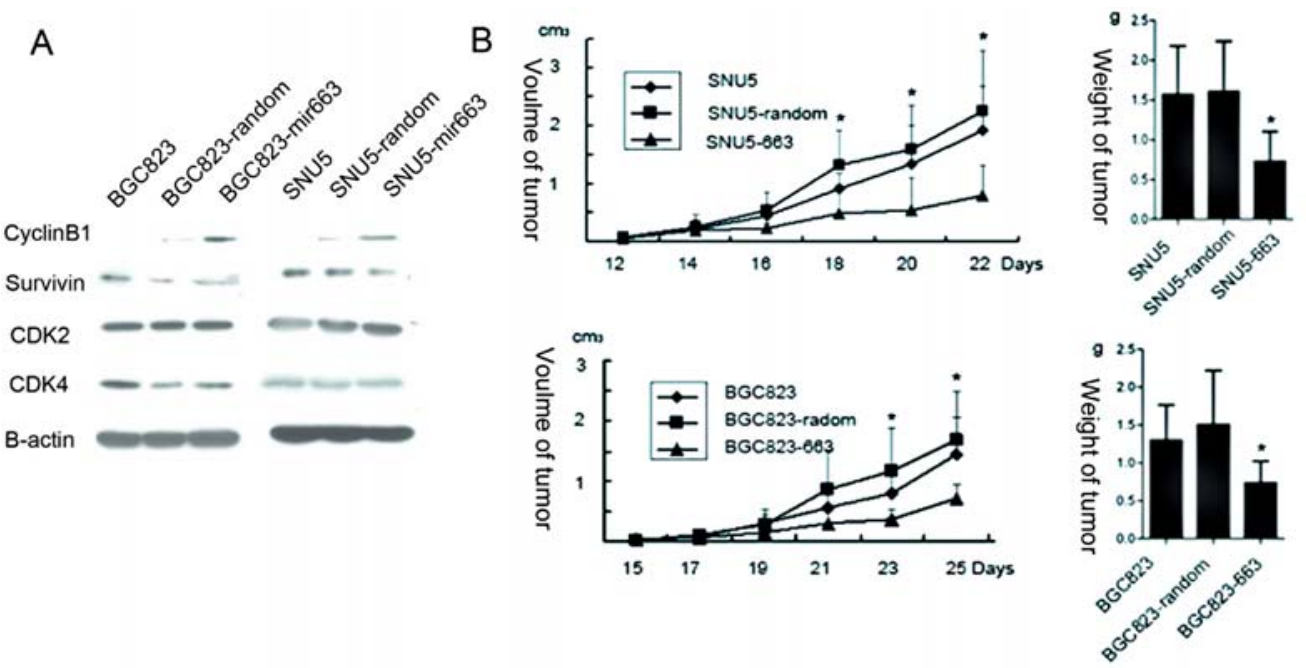

C
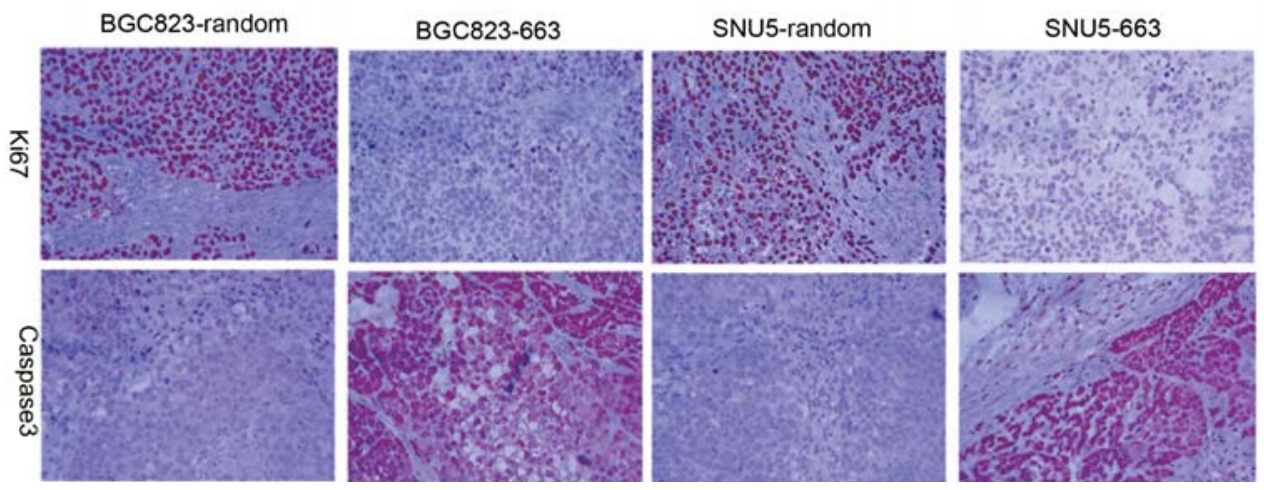

Figure 4. Administration of mir-663 suppresses tumor growth in vivo. (a) Western blot analysis of the expression of proteins in human gastric cancer cells BGC823 and SNU5 transferred with mir-663. (b) Relative cell growth was measured at the indicated times. Mir-663 significantly inhibited the growth of these cells. The values are the means from 6 animals \pm SD. (c) Immunohistochemical analysis of mir-663 treated group with Ki67 and caspase-3 antibody. At least five areas of each section were examined at a magnification of x200.

mitotic index slides that had several nuclear envelopes, but no obvious chromosomes were present (upper panel of Fig. 3A and B). These structures were neither interphase nuclei nor chromosomal spreads; they were more clearly visible when the cells were stained with DAPI and $\alpha$-tubulin (lower panel of Fig. 3A and B). These structures were present in high numbers only in mir-663-treated cells. We speculated that these were mitotic cells undergoing mitotic arrest, which would explain the 'sub-G2' population indicated in the flow cytometry data. These structures are likely indicators of mitotic catastrophe, which has been shown to occur in cells lacking cell cycle checkpoints such as loss of p53 (21). The effect of mir-663 induction on mitotic catastrophe was examined. Cells had a significantly higher mitotic catastrophe index in the mir-663 group (abnormal nuclear cells, about 250/1000) than in the parent and random groups (abnormal nuclear cells, about 30/1000) (right panel of Fig. 3A and B). The chromosome analysis showed that the chromosome number of the BGC823 and SNU5 cells transfected with mir-663 ranged from $<40$ to $>140$, and that of cells in the control group was mostly about 46 (Fig. 3C and D). These results confirmed that mir-663 can cause mitotic catastrophe in gastric carcinoma cells.

Protein markers of mitotic catastrophe. The cells were transfected with mir-663 and analyzed by Western blot after $72 \mathrm{~h}$ for protein markers of mitotic arrest and mitotic catastrophe (Fig. 4A). The levels of cyclin B were elevated to a greater extent in mir-663-transfected cells than in the control cells. Cyclin $\mathrm{B} /$ cell division cycle $2(C D C 2)$ activity is essential for entering mitosis. In contrast, mitotic exit requires cyclin $\mathrm{B} / C D C 2$ suppression, which normally occurs due to the proteasomal degradation of cyclin $\mathrm{B}$ after ubiquitination by the anaphase-promoting complex/CDH1 complex (22-24). Therefore, cyclin B is very important for the natural cell cycle. Protein of surviving CDK2 and CDK4 had little change with mir-663 (Fig. 4A).

Gene therapy with mir-663 suppresses the growth of gastric cancer cells in vivo. The engrafted tumors were treated with mir-663 mimic RNA, which was introduced by liposomemediated gene delivery. The mice were sacrificed 30 days post-injection. The tumor growth was significantly suppressed by the treatment with mir-663 mimic RNA (Fig. 4B). The tumor burden was reduced by $50 \%$ after 30 days of injection. The average tumor volumes decreased significantly with the administration of mir-663 as compared with those that were administered control RNA throughout the entire experimental period of about 30 days (Fig. 4B). Organs, including the heart, lung, liver, kidney, spleen, and enteron, were subjected to histological analyses. No obvious systemic side effects were observed in the organs of the treated mice. The 
average mouse weight, excluding the tumor weight, of the treated and control groups were not significantly different when the treatment ended. The tumor tissues treated with mir-663 showed a considerable number of necrotic areas and a significant decrease in $\mathrm{Ki} 67$ and increase in caspase-3 immunohistochemistry staining as compared with those treated with control RNA (Fig. 4C). These results indicate that the introduction of mir-663 suppresses the growth of human gastric cancer cells in an in vivo setting as well.

\section{Discussion}

The discovery of the role of miRNAs in various pathological processes has opened up the possibility of using miRNAs in molecular diagnostics and prognostics, particularly in the case of cancer. In our study, mir-663 was identified as a mitotic catastrophe gene in gastric cancer cell lines; this gene almost completely arrested cell proliferation. This miRNA had low expression levels in all gastric cancer cell lines examined by us. Therefore, we concluded that the silencing of mir-663 may be a common event in gastric cancer. The introduction of mir-663 into two gastric cancer cell lines, BGC823 and SNU5, showed strong inhibition of cell proliferation, and a mitotic catastrophe accompanied by the upregulation of cyclin $\mathrm{B}$.

In recent years, the expression 'mitotic catastrophe' has been widely used to describe a form of death of mammalian cells. Roninson attempted to define 'mitotic catastrophe' in morphological terms as a type of cell death resulting from abnormal mitosis, which usually ends in the formation of large cells with multiple micronuclei and decondensed chromatin (26). The Cdk1/cyclin B complex is essential for mitosis and mitotic catastrophe. Cdk1 interacts with its obligate allosteric activator-cyclin B to form an active heterodimer, the 'mitosis-promoting factor'. The progression from the $\mathrm{G} 2$ to $\mathrm{M}$ phase is driven by the activation of the $\mathrm{Cdk} 1 /$ cyclin B complex, whose activity must be sustained from prophase to metaphase (23). Elevated levels of cyclin B have been found in numerous studies on mitotic catastrophe induced by pharmacological or genetic manipulations. For instance, increase in level of cyclin B was found in human colorectal adenocarcinoma cell lines treated with fluorouracil and 14-3-3s-/- colon cancer cells treated with doxorubicin (27).

For miRNAs whose expression is reduced in the disease state, the reintroduction of the mature miRNAs into the proper tissue could provide a therapeutic benefit by restoring the regulation of target genes, since chemicals, heat, and/or ionizing radiation used as tumor suppressors in the treatment of cancer often induce mitotic catastrophe (21). We attempted to estimate the effect of mir-663 in cancer treatment. Cationic liposomes have been used successfully for gene transfer in multiple organ systems (28). The ease of preparation and administration, low toxicity and immunogenicity, as well as the ability to transfer large fragments of insert DNA have resulted in their use as alternative vehicles for gene delivery (29). Our experiments showed that cationic liposomemediated mir-663 delivery can significantly suppress the growth of gastric cancer cells in vivo. Recently, McCaffrey and coworkers found that sustained, high-level short hairpin
RNA (shRNA) expression produced lethal, dose-dependent liver injury (30). An individual miRNA may post-tanscriptionally regulate over hundreds of different mRNAs, therefore, miRNAs may also have off-target effects and damage normal tissues. Our experiment showed that mir-663 has little effect on the weight of the mice, and their organs had no abnormality. Therefore, introducing mir-663 into tumors might be a promising targeting treatment for cancer. In addition, some miRNAs are reported to be controlled by epigenetic alterations in cancer cells, including DNA methylation and histone modification. Target oncogenes can be regulated by using chromatin-modifying drugs to activate tumor suppressor miRNA; this knowledge can be used to develop novel cancer therapies in the future $(31,32)$. Since mir-663 has been reported to be downregulated because of aberrant hypermethylation in cancer, we think that the de-hypermethylation of mir-663 might lead to a powerful anticancer treatment.

The mechanism of mir-663-mediated suppression of gastric cancer cells might be related to the direct modulation of other downstream targets. Although the messenger RNA targets of mir-663 are not known, the identification and validation of such targets could be useful for determining the potential value of the delivery of mir-663 into gastric cancer cells as a cancer-specific therapeutic strategy. Our data suggest that mir-663 may hold significant promise as a novel target in molecular therapy for human gastric cancer.

\section{Acknowledgments}

This study was supported by grants from the National Key Basic Research Program (NKBRP) (973 program) (No. 2009CB521804) and the National Natural Science Foundation (30570818 and 30600279).

\section{References}

1. Tong AW and Nemunaitis J: Modulation of miRNA activity in human cancer: a new paradigm for cancer gene therapy? Cancer Gene Ther 15: 341-355, 2008.

2. Peng Y, Laser J, Shi G, Mittal K, Melamed J, Lee P and Wei JJ: Antiproliferative effects by Let-7 repression of high-mobility group A2 in uterine leiomyoma. Mol Cancer Res 6: 663-673, 2008.

3. Yan D, Zhou X, Chen X, Hu D, Dong XE, Wang J, Lu F, Tu L and Qu J: MicroRNA-34a inhibits uveal melanoma cell proliferation and migration through downregulation of c-Met. Invest Ophthalmol Vis Sci 50: 1559-1565, 2009.

4. Papagiannakopoulos T, Shapiro A and Kosik KS: MicroRNA21 targets a network of key tumor-suppressive pathways in glioblastoma cells. Cancer Res 68: 8164-8172, 2008.

5. Shi L, Cheng Z, Zhang J, Li R, Zhao P, Fu Z and You Y: hsamir-181a and hsa-mir-181b function as tumor suppressors in human glioma cells. Brain Res 1236: 185-193, 2008.

6. Bonci D, Coppola V, Musumeci M, Addario A, Giuffrida R, Memeo L, D'Urso L, Pagliuca A, Biffoni M, Labbaye C, Bartucci M, Muto G, Peschle C and De Maria R: The miR-15a-miR-16-1 cluster controls prostate cancer by targeting multiple oncogenic activities. Nat Med 14: 1271-1277, 2008.

7. Guglielmelli P, Tozzi L, Pancrazzi A, Bogani C, Antonioli E, Ponziani V, Poli G, Zini R, Ferrari S, Manfredini R, Bosi A and Vannucchi AM: MicroRNA expression profile in granulocytes from primary myelofibrosis patients. Exp Hematol 35: 1708-1718, 2007.

8. Marsh EE, Lin Z, Yin P, Milad M, Chakravarti D and Bulun SE: Differential expression of microRNA species in human uterine leiomyoma versus normal myometrium. Fertil Steril 89: 1771-1776, 2008. 
9. Volinia S, Calin GA, Liu CG, Ambs S, Cimmino A, Petrocca F Visone R, Iorio M, Roldo C, Ferracin M, Prueitt RL, Yanaihara N, Lanza G, Scarpa A, Vecchione A, Negrini M, Harris CC and Croce CM: A microRNA expression signature of human solid tumors defines cancer gene targets. Proc Natl Acad Sci USA 103: 2257-2261, 2006

10. Marton S, Garcia MR, Robello C, Persson H, Trajtenberg F, Pritsch O, Rovira C, Naya H, Dighiero G and Cayota A: Small RNAs analysis in CLL reveals a deregulation of miRNA expression and novel miRNA candidates of putative relevance in CLL pathogenesis. Leukemia 22: 330-338, 2008.

11. Bottoni A, Piccin D, Tagliati F, Luchin A, Zatelli MC and degli Uberti EC: miR-15a and miR-16-1 down-regulation in pituitary adenomas. J Cell Physiol 204: 280-285, 2005.

12. Tokumaru S, Suzuki M, Yamada H, Nagino M and Takahashi T: let-7 regulates Dicer expression and constitutes a negative feedback loop. Carcinogenesis 29: 2073-2077, 2008.

13. Toyota M, Suzuki H, Sasaki Y, Maruyama R, Imai K, Shinomura $\mathrm{Y}$ and Tokino T: Epigenetic silencing of micro$\mathrm{RNA}-34 \mathrm{~b} / \mathrm{c}$ and B-cell translocation gene 4 is associated with $\mathrm{CpG}$ island methylation in colorectal cancer. Cancer Res 68: 4123-4132, 2008

14. Grady WM, Parkin RK, Mitchell PS, Lee JH, Kim YH, Tsuchiya KD, Washington MK, Paraskeva C, Willson JK, Kaz AM, Kroh EM, Allen A, Fritz BR, Markowitz SD and Tewari M: Epigenetic silencing of the intronic microRNA hsa-miR-342 and its host gene EVL in colorectal cancer. Oncogene 27: 3880-3888, 2008.

15. Lehmann U, Hasemeier B, Christgen M, Muller M, Romermann D, Langer F and Kreipe H: Epigenetic inactivation of microRNA gene hsa-mir-9-1 in human breast cancer. J Pathol 214: 17-24, 2008

16. Rokhlin OW, Scheinker VS, Taghiyev AF, Bumcrot D, Glover RA and Cohen MB: MicroRNA-34 mediates ARdependent $\mathrm{p} 53$-induced apoptosis in prostate cancer. Cancer Biol Ther 7: 1288-1296, 2008.

17. Zhu S, Wu H, Wu F, Nie D, Sheng S and Mo YY: MicroRNA21 targets tumor suppressor genes in invasion and metastasis. Cell Res 18: 350-359, 2008.

18. Castedo M, Perfettini JL, Roumier T, Yakushijin K, Horne D, Medema R and Kroemer G: The cell cycle checkpoint kinase Chk2 is a negative regulator of mitotic catastrophe. Oncogene 23: 4353-4361, 2004

19. Ianzini F and Mackey MA: Delayed DNA damage associated with mitotic catastrophe following X-irradiation of HeLa S3 cells. Mutagenesis 13: 337-344, 1998 .

20. Daftary GS and Taylor HS: Efficient liposome-mediated gene transfection and expression in the intact human uterus. Hum Gene Ther 12: 2121-2127, 2001.
21. Castedo M and Kroemer G: Mitotic catastrophe: a special case of apoptosis. J Soc Biol 198: 97-103, 2004.

22. Niida H, Tsuge S, Katsuno Y, Konishi A, Takeda N and Nakanishi M: Depletion of Chk1 leads to premature activation of Cdc2-cyclin B and mitotic catastrophe. J Biol Chem 280: 39246-39252, 2005.

23. Scaife RM: G2 cell cycle arrest, down-regulation of cyclin B, and induction of mitotic catastrophe by the flavoprotein inhibitor diphenyleneiodonium. Mol Cancer Ther 3: 1229-1237, 2004.

24. Michel L, Diaz-Rodriguez E, Narayan G, Hernando E, Murty VV and Benezra R: Complete loss of the tumor suppressor MAD2 causes premature cyclin B degradation and mitotic failure in human somatic cells. Proc Natl Acad Sci USA 101: 4459-4464, 2004.

25. Mackey D and Sugden B: Studies on the mechanism of DNA linking by Epstein-Barr virus nuclear antigen 1. J Biol Chem 272: 29873-29879, 1997.

26. Roninson IB, Broude EV and Chang BD: If not apoptosis, then what? Treatment-induced senescence and mitotic catastrophe in tumor cells. Drug Resist Updat 4: 303-313, 2001.

27. Chan CC, Boyce S, Brideau C, et al: Rofecoxib [Vioxx, MK0966; 4-(4'-methylsulfonylphenyl)-3-phenyl-2-(5H)-furanone]: a potent and orally active cyclooxygenase- 2 inhibitor. Pharmacological and biochemical profiles. J Pharmacol Exp Ther 290: $551-560,1999$

28. Kikuchi M, Ohsaka A, Chiba Y, Sato M, Muraosa Y and Hoshino H: Bone marrow aplasia with prominent atypical plasmacytic proliferation preceding acute lymphoblastic leukemia. Leuk Lymphoma 35: 213-217, 1999.

29. Garay EB and Rutkin AS: Avoiding the legal pitfalls of testimonials and endorsements. J Healthc Risk Manag 17: 7-12, 1997.

30. McCaffrey AP, Nakai H, Pandey K, Huang Z, Salazar FH, $\mathrm{Xu} \mathrm{H}$, Wieland SF, Marion PL and Kay MA: Inhibition of hepatitis B virus in mice by RNA interference. Nat Biotechnol 21: 639-644, 2003.

31. Reber S, Over S, Kronja I and Gruss OJ: CaM kinase II initiates meiotic spindle depolymerization independently of APC/C activation. J Cell Biol 183: 1007-1017, 2008.

32. Yoshikawa R, Kusunoki M, Yanagi H, Noda M, Furuyama JI, Yamamura $\mathrm{T}$ and Hashimoto-Tamaoki T: Dual antitumor effects of 5-fluorouracil on the cell cycle in colorectal carcinoma cells: a novel target mechanism concept for pharmacokinetic modulating chemotherapy. Cancer Res 61: 1029-1037, 2001. 